\title{
ATHEROSCLEROSIS IN MONOGENIC FAMILIAL HYPERCHOLESTEROLAEMIA VERSUS POLYGENIC HYPERCHOLESTEROLAEMIA
}

\author{
Mahtab Sharifi ${ }^{1,2}$, Angela Gallivan ${ }^{2}$, Darren Harvey ${ }^{2}$, Jackie Cooper ${ }^{1}$, Ka Wah Li $^{1}$, Martha \\ Futema ${ }^{1}$, Steve E. Humphries ${ }^{1}$, Devaki Nair ${ }^{2}$ \\ ${ }^{1}$ Centre for Cardiovascular Genetic, University College London, The Rayne Institute, \\ University Street, London, UK \\ ${ }^{2}$ Department of Clinical Biochemistry, Royal Free London NHS Foundation Trust, Pond \\ Street, London, UK
}

Introduction: The aim of this study was to assess the degree of atherosclerosis in monogenic Familial Hypercholesterolaemia (FH) patients versus individuals with a clinical diagnosis of FH but with no detectable mutation (ie with polygenic hypercholesterolaemia).

Method: Carotid Intima Media Thickness (cIMT) was measured by B-mode ultrasound in the common carotid artery, bifurcation and internal carotid artery in 86 individuals ( 53 females and 33 male) with a clinical diagnosis of FH (LDL-C mean+SD: $5.8 \pm 1.3 \mathrm{mmol} / \mathrm{l}) .56$ patients had monogenic $\mathrm{FH}$ with a mutation in the $\angle D L R$ or $A P O B$ gene and 30 patients had no mutation and had a score in the top two quartiles of a six LDL-C-raising SNPs gene score.

Results: The monogenic patients were younger than polygenic individuals ( $50 \pm 14$ years vs $57 \pm 12$ years, $p=0.03$ ). There was no significant difference in total cholesterol level, LDL-C and HDL-C between the two groups. Triglyceride level was significantly higher in the polygenic compared to the monogenic group $(1.6 \pm 0.7 \mathrm{mmol} / \mathrm{l}$ vs $1.2 \pm 0.5 \mathrm{mmol} / \mathrm{l}, \mathrm{p}=0.01]$. After adjustment for age and gender, the mean of all the cIMT measurements was significantly higher in monogenic than polygenic patients $[0.74 \mathrm{~mm}(0.7-0.79)$ vs $0.66 \mathrm{~mm}$ (0.61-0.72), $p=0.039]$.

Conclusion: The severity of atherosclerosis as measured by $\mathrm{CIMT}$ is higher in monogenic $\mathrm{FH}$ individuals than the polygenic group. While LDL-C levels need to be reduced in both groups, the greater degree of carotid atherosclerosis supports aggressive management of their LDL$C$ levels with potent statins and other LDL-C lowering modalities in combination. 